\title{
High-fat diet induces lung remodeling in ApoE-deficient mice: an association with an increase in circulatory and lung inflammatory factors
}

\author{
Amarjit S Naura ${ }^{1}$, Chetan P Hans ${ }^{1}$, Mourad Zerfaoui ${ }^{1}$, Youssef Errami ${ }^{1}$, Jihang Ju${ }^{2}$, Hogyoung Kim², \\ Khalid Matrougui ${ }^{3}$, Jong $\mathrm{G} \mathrm{Kim}{ }^{2,4}$ and A Hamid Boulares ${ }^{1}$
}

Hypercholesterolemia is increasingly considered the basis for not only cardiovascular pathologies but also several complications affecting other organs such as lungs. In this study, we examined the effect of hypercholesterolemia on lung integrity using a mouse model $\left(\mathrm{ApoE}^{-/-}\right)$of high-fat $(\mathrm{HF})$ diet-induced atherosclerosis. A 12-week $\mathrm{HF}$ diet regimen induced systemic production of TNF- $\alpha$, IFN- $\gamma$, GMC-SF, RANTES, IL- $1 \alpha$, IL-2 and IL-12 with TNF- $\alpha$ as the predominant cytokine in $\mathrm{ApoE}^{-1-}$ mice. Concomitantly, TNF- $\alpha$, IFN- $\gamma$ and MIP- $1 \alpha$ were detected in brochoalveolar lavage (BAL) fluids of these mice, coinciding with lung inflammation consisting primarily of monocytes/macrophages. Such lung inflammation correlated with marked collagen deposition and an increase in matrix metalloproteinase- 9 activity in ApoE ${ }^{-1}$ mice $^{-}$ without mucus production. Although TGF- $\beta 1$ was undetectable in the BAL fluid of $\mathrm{ApoE}^{-1-}$ mice on $\mathrm{HF}$ diet, it showed a much wider tissue distribution compared with that of control animals. Direct exposure of smooth muscle cells to oxidized-LDL, in vitro, induced a time-dependent expression of TNF- $\alpha$. Direct intratracheal TNF- $\alpha$-administration induced a lung inflammation pattern in wild-type mice that was strikingly similar to that induced by HF diet in $\mathrm{ApoE}^{-/-}$mice. TNF- $\alpha$ administration induced expression of several factors known to be critically involved in lung remodeling, such as MCP-1, IL- $1 \beta$, TGF- $\beta 1$, adhesion molecules, collagen type-I and TNF- $\alpha$ itself in the lungs of treated mice. These results suggest that hypercholesterolemia may promote chronic inflammatory conditions in lungs that are conducive to lung remodeling potentially through TNF- $\alpha$-mediated processes.

Laboratory Investigation (2009) 89, 1243-1251; doi:10.1038/labinvest.2009.98; published online 14 September 2009

KEYWORDS: lung remodeling; atherosclerosis; cytokines; inflammation; $\mathrm{ApoE}^{-1-}$ mouse

The etiology of many diseases can be linked to diet or changes in the diet. A high-fat (HF) diet is increasingly regarded as a primary cause for numerous diseases such as diabetes, hypertension and cancer. ${ }^{1}$ The relationship between dietary fat and the risk for cardiovascular diseases has been extensively examined. A direct link between diets high in saturated and trans fatty acids increases LDL cholesterol levels, and the increased risk of heart disease has been well established. ${ }^{2}$ Despite evidence for the involvement of a HF diet in inflammatory diseases, few studies have been undertaken to examine the involvement of a HF in lung inflammation. More recently, hypercholesterolemia was reported to be a potential risk factor for asthma in humans. ${ }^{3-5}$
Remodeling occurs in a number of chronic lung inflammatory diseases. ${ }^{6,7}$ Chronic inflammation is thought to initiate and perpetuate cycles of tissue injury and repair in asthma, although remodeling may also occur in parallel with inflammation. The persistence of inflammatory cells within the airways and the upregulation of a number of inflammatory cytokines and growth factors such as tumor necrosis factor (TNF), tumor growth factor beta (TGF- $\beta$ ) and several members of the interleukin family, as well as adhesion molecules, participate in the onset of lung remodeling. ${ }^{7}$

Given that HF diet may increase the production of inflammatory factors in the circulation, and the fact that

\footnotetext{
${ }^{1}$ Department of Pharmacology and Experimental Therapeutics, Louisiana State University Health Sciences Center, New Orleans, LA, USA; ${ }^{2}$ Department of Pathology, Louisiana State University Health Sciences Center, New Orleans, LA, USA and ${ }^{3}$ Department of Physiology, Tulane University Medical Center, New Orleans, LA, USA Correspondence: Dr AH Boulares, PhD, Department of Pharmacology and Experimental Therapeutics, Louisiana State University Health Sciences Center, 1901 Perdido Street, New Orleans, LA 70112, USA.

E-mail: hboulr@|suhsc.edu

${ }^{4}$ Current address: Department of Animal Science and Biotechnology, Chonbuk National University, Jeonju, Korea

Received 24 April 2009; revised 16 July 2009; accepted 3 August 2009
} 
airways come into contact with circulatory elements in a continuous manner, we hypothesized that inflammatory factors may influence pathological events within the lungs. To test this, we used $\mathrm{ApoE}^{-1-}$ mice, which is a well-established animal model of HF diet-induced chronic inflammation. Our results suggest that HF diet promotes chronic inflammation in the circulation that may influence critical changes within the lungs, leading to lung remodeling and ultimately to further lung complications.

\section{MATERIALS AND METHODS}

\section{Animals, Diet and Treatment Protocols}

Wild-type and $\mathrm{ApoE}^{-1-}$ mice were purchased from Jackson Laboratories (Bar Harbor, ME, USA). Mice were bred in a specific-pathogen free facility at LSUHSC, New Orleans, LA, USA and allowed unlimited access to sterilized chow and water. Maintenance and experimental protocols were all approved by the LSUHSC Animal Care \& Use Committee. C57BL/6 6-week-old $\mathrm{ApoE}^{-/-}$mice received regular chow or a HF diet (Harlan Teklad, Madison, WI, USA) containing $21 \%$ fat by weight $(0.15 \%$ cholesterol) for 12 weeks before killing. Wild-type mice were administered TNF- $\alpha$ (100 ng/mouse) intratracheally under anesthesia. Mice were killed 6 or $24 \mathrm{~h}$ later for lung collection or brochoalveolar lavage (BAL) fluids, respectively. As a control for IL-13 production, 6-week-old C57BL/6 wild-type mice were sensitized and challenged with ovalbumin as described earlier. ${ }^{8,9}$

\section{Organ Recovery and Tissue Staining}

Mice were fasted, anesthetized with ketamine/xylazine (60 and $3 \mathrm{mg} / \mathrm{kg}$, respectively), and blood was drawn for sera preparation. Animals were killed and the aortas or lungs were isolated as described. ${ }^{8-10}$ Lungs were fixed with $10 \%$ formalin and processed for pathology and immunohistochemistry (IHC) analysis, immersed in RNA Later solution (Qiagen Inc., Valencia, CA, USA) for RNA extraction, or subjected to BAL. Formalin-fixed lungs were sectioned and subjected to hematoxylin and eosin $(\mathrm{H} \& \mathrm{E})$, trichrome or Periodic Acid-Schiff (PAS) staining using standard protocols. IHC was conducted as described earlier ${ }^{11}$ using antibodies to murine transforming growth factor- $\beta$ (TGF- $\beta$ ) (Cell Signaling Technology Inc., Beverly, MA, USA). BAL fluids were used for cytokine measurements or subjected to cyto-spin and stained with H\&E for the assessment of inflammatory cells.

Perfusion-fixed aortas were dissected and prepared for either Oil-Red-O staining using standard protocol or paraffin embedding for H\&E staining. Formalin-fixed hearts were incubated in $5 \%$ and then $10 \%$ gelatin solutions for $2 \mathrm{~h}$ each, followed by an overnight incubation in $25 \%$ gelatin. Serial sections were obtained from aortic sinus after embedding in OCT media and stained with Oil-Red-O as described. ${ }^{10}$

\section{Isolation of Primary Smooth Muscle Cells (SMCs) from Mouse Lung}

SMCs were isolated from C57BL/6 mice as described earlier ${ }^{10}$ after adaptation of lung tissue as described by Amrani et al. ${ }^{12}$ SMCs, at passages $3-5$, were grown to $80 \%$ confluency in $10 \%$ FBS-DMEM and then growth arrested for $24 \mathrm{~h}$ in serum-free DMEM before stimulation with $100 \mu \mathrm{g} / \mathrm{ml}$ oxidized cholesterol (oxLDL) for 3 or $6 \mathrm{~h}$. Cells were then collected for RNA analysis by real-time PCR.

\section{Bioplex Assay for Cytokine Measurement}

Serum or BAL fluids were used for assessment of different cytokines using the Bio-Rad single-plex or multiplex assay kits (Bio-Rad Laboratories, Hercules, CA, USA) as per the instructions of the manufacturer and as described. ${ }^{9}$ Serum samples were processed for removal of lipids using lipoclear reagent (Iris Sample Processing, Westwood, MA, USA) before testing according to the manufacturer's instructions. (According to Bio-Rad Laboratories, cross-reactivity between different cytokines in the utilized multiplex system is negligible. In addition, intraassay and inter-assay coefficients of variation are noted to be minimal. http://www.bio-rad.com/ cmc_upload/Literature/199110/Bulletin_3157.pdf).

\section{Reverse Transcription, PCR and Gelatin Zymography}

RNA was extracted from lung tissue and cDNA was generated by standard methods. The set of primers used for different genes are described in Table 1 (Supplementary Material). The specificity of the primer sets was confirmed in our earlier studies. ${ }^{10,13,14}$ Amplification, detection and data analysis were performed using the iCycler PCR system (Bio-Rad Laboratories). For zymography, protein extracts were prepared by homogenizing lungs in lysis buffer $(1: 4 \mathrm{w} / \mathrm{v})^{15}$ and were $(50 \mu \mathrm{g})$ immediately assayed for matrix metalloproteinase (MMP) activity with SDS-PAGE gelatin using commercial gels (Invitrogen, Carlsbad, CA, USA) according to the manufacturer's instructions.

\section{Statistical Analysis}

All data are expressed as mean \pm s.d. of values from three independent experiments having at least six mice per group. PRISM software (GraphPad, San Diego, CA, USA) was used to analyze the differences between experimental groups by one-way ANOVA. $P$-values of $<0.05$ were considered significant.

\section{RESULTS}

Systemic Production of Inflammatory Cytokines That have the Potential to Affect Lung Integrity in $\mathrm{ApoE}^{-1-}$ Mice on a High-Fat Diet

Figure 1a shows that subjecting $\mathrm{ApoE}^{-/-}$mice to a HF diet for 12 weeks promoted the marked formation of atherosclerotic plaques, as expected. The plaques showed cholesterol-rich lipid clefts within the intimal layer containing 
a

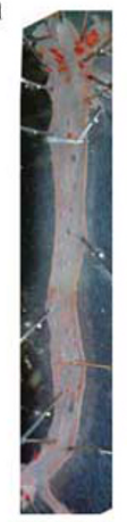

Reg Diet

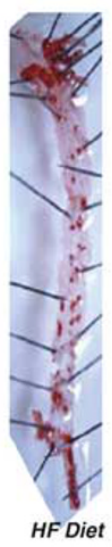

d

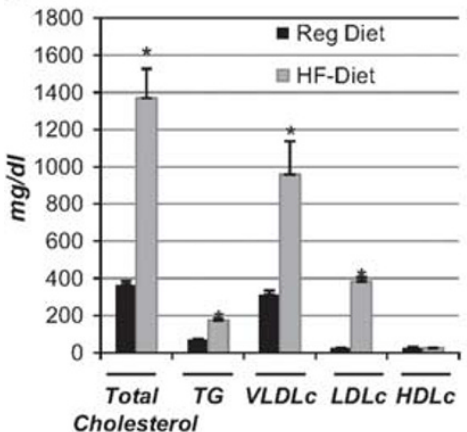

b
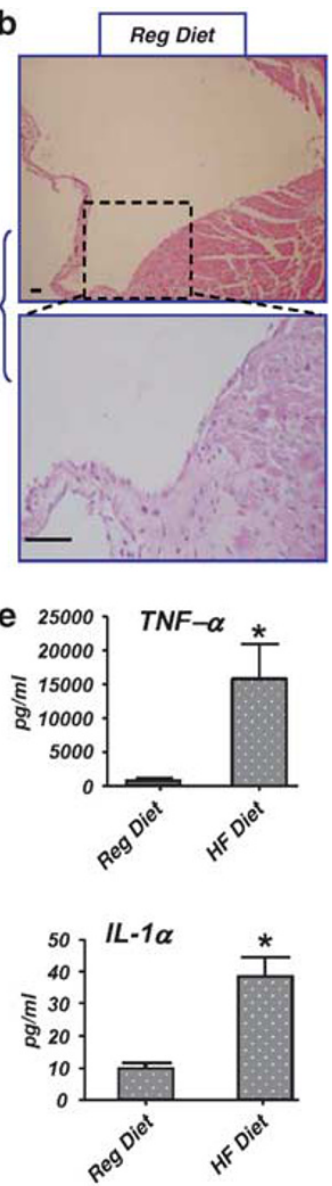
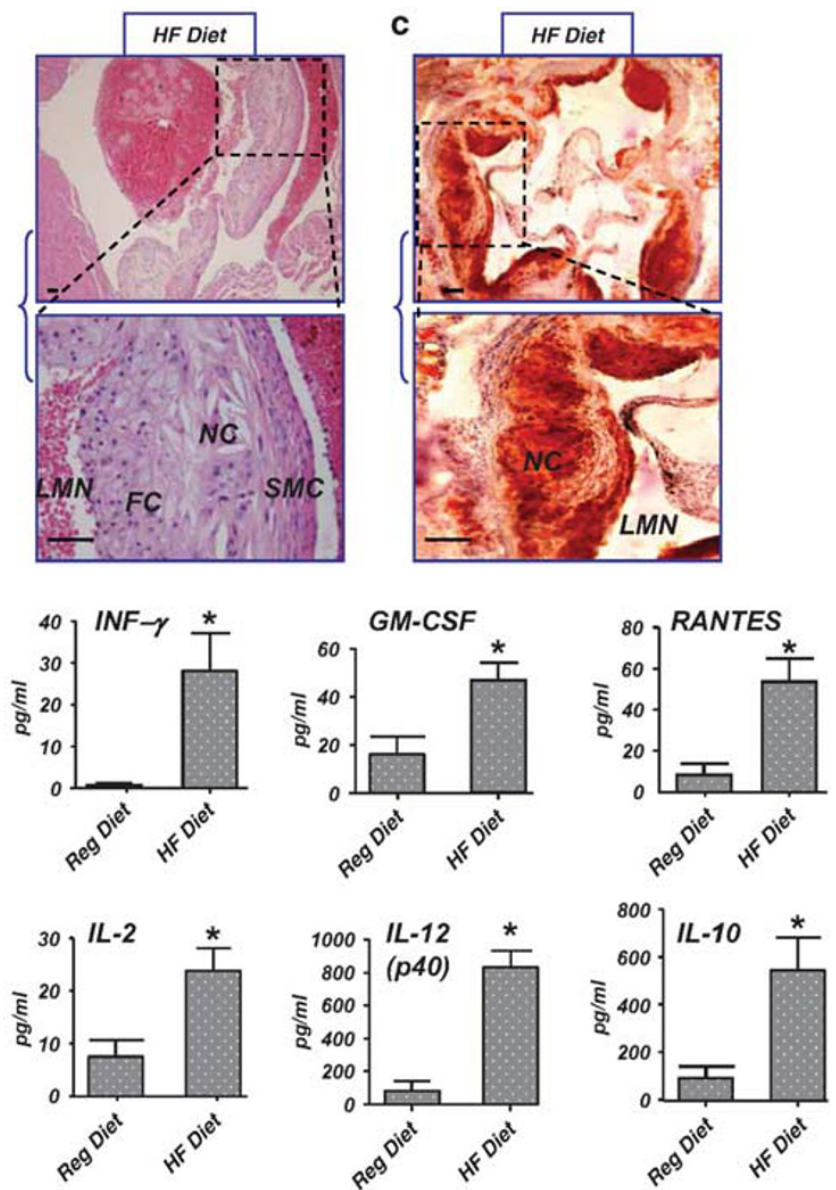

Figure 1 High-fat (HF) diet induction of atherogenesis is correlated with substantial systemic inflammatory cytokine production in ApoE ${ }^{-/-}$mice. $\mathrm{C}^{2} \mathrm{BL} / 6$ $\mathrm{ApoE}^{-/-}$mice were fed either high-fat (HF diet) or regular (Reg diet) diet for 12 weeks, after which mice were killed. (a) Aortas were formalin (PBS)-perfused and atherosclerotic plaques were visualized by en face Oil-Red-O staining. (b) Aortic sinus sections were prepared as described in Materials and methods, subjected to hematoxylin and eosin (H\&E) staining and visualized by light microscopy. Abbreviations: FC, foam cells; NC, necrotic core; LMN, lumen; SMC, smooth muscle cells. (c) Aortic sinus sections from HF diet-fed mice were subjected to Oil-Red-O staining to depict lipid deposits within atherosclerotic plaques; bar: $5 \mu \mathrm{m}$. (d) Mice from the different experimental groups were fasted, anesthetized, and blood was drawn for sera preparation. Plasma cholesterol and LDL (mg/dl) were analyzed; $n=4$. ${ }^{*}$ Difference from $\mathrm{ApoE}^{-/-}$mice fed a regular diet for 12 weeks; $P<0.01$. (e) Sera were collected and cytokines were assessed using a multi-panel Bio-Rad Bioplex system as described. ${ }^{9}$ Data are given as means \pm s.d. of values obtained from at least six mice per group. ${ }^{*}$ Difference from $\mathrm{ApoE}^{-1-}$ mice fed a regular diet; $P<0.01$. Please note that the graphs are based on different scales.

distinct, large macrophage-derived foam cells and SMCs (Figure 1b). Figure 1c depicts the lipid-rich nature of the atherosclerotic plaques at the aortic sinus after staining with Oil-Red-O. Serum cholesterol and triglyceride levels were significantly higher in $\mathrm{ApoE}^{-1-}$ mice on a HF diet compared with those in mice on a regular diet (data not shown), which is consistent with our earlier reported data, ${ }^{10}$ as well as with many published reports (for a review, see Libby ${ }^{16}$ ). Serum cholesterol and triglycerides were measured to evaluate the effect of HF diet on the lipid profile of the experimental model. Figure 1d shows that the HF atherogenic diet significantly increased serum TG, TC, LDL-C and HDL-C levels, which is consistent with published reports. ${ }^{10}$

An assessment of the major cytokines produced after 12 weeks of the HF diet (Figure 1d) reveals a significant elevation in the production of TNF- $\alpha$, IFN- $\gamma$, GMC-SF, RANTES,
IL- $1 \alpha$, IL-2 and IL-12 compared with serum levels of animals that were fed a regular diet. The anti-inflammatory cytokine IL-10 was also elevated to levels similar to those of the proatherogenic cytokine IL-12 in response to the HF diet in ApoE ${ }^{-1-}$ mice. A number of other cytokines, such as MIP- $1 \alpha$, keratinocyte-derived cytokine (KC), IL-17, G-CSF and IL-6, were also measured, but no significantly increased levels were detected in the HF diet group (data not shown). Given that lungs can be exposed to systemic factors because of their significant exchange with the vasculature, and the fact that the roles of the cytokines induced by the HF diet, in our experimental model, in lung remodeling are well established (for a review, see Vignola et al. ${ }^{17}$ ), we surmised that the HF diet may promote cytokine and growth factor elevation that could create chronic inflammatory conditions conducive to lung remodeling. 


\section{The High-Fat Diet Induces Production of TNF- $\alpha$, IFN- $\gamma$ and MIP-1 $\alpha$ in Lungs of ApoE ${ }^{-1-}$ Mice}

To test the potential effect of a HF diet on lung homeostasis and integrity, we assessed different cytokine levels in our animal model. Figure 2 shows that varying levels of TNF- $\alpha$, IFN- $\gamma$ and MIP- $1 \alpha$ were present in the BAL fluid of HF dietfed $\mathrm{ApoE}^{-1-}$ mice compared with those of mice receiving regular diet. However, no major differences were observed in the levels of GMC-SF, IL- $1 \alpha$, IL-12 or IL-10 between the two experimental groups (data not shown); IL-2 and RANTES were undetectable in both experimental groups (data not shown). The reasons behind the observed selective cytokine elevation in the lungs are not clear.

Deletion of the ApoE gene has been shown to promote inflammatory conditions. Accordingly, ApoE deficiency may represent a confounding factor on whether high lipid levels would directly affect lung homeostasis. To analyze whether high cholesterol levels could promote an inflammatory response in lung cells, we examined whether a direct exposure of lung SMCs to oxLDL would culminate in an induction of TNF- $\alpha$ expression. Figure $2 \mathrm{~b}$ shows that oxLDL induced a significant increase in TNF- $\alpha$ expression and in a time-dependent manner as assessed by real-time PCR. This result clearly suggests that lung cells can be susceptible to elevated cholesterols and that the latter may produce inflammatory conditions in the lung.

\section{Association between Pro-Inflammatory Cytokines in BAL Fluid from High-Fat Diet-Fed ApoE $^{-1-}$ Mice and Lung Inflammation Consisted Primarily of Monocytes/ Macrophages}

The roles of TNF- $\alpha$, IFN- $\gamma$ and MIP- $1 \alpha$ in lung pathologies have been reported in numerous studies (reviewed in Broide $^{7}$ ). To determine the consequences of elevated cytokine production, we assessed the histopathology of lungs from the two experimental groups. H\&E staining of lung sections from the HF diet-fed $\mathrm{ApoE}^{-/-}$mice revealed a substantial recruitment of inflammatory cells, mostly macrophages/ monocytes (Figure 3a), which was largely absent in the $\mathrm{ApoE}^{-1-}$ mice that received a regular diet. Figure $3 \mathrm{~b}$ confirms the macrophage/monocytic nature of the recruited inflammatory cells by subjecting BAL fluid to a cytospin followed by H\&E staining. Additional support for the identity of macrophages/monocytes was obtained by immunofluorescence with antibodies to CD68 (Figure 3b, bottom panel). Figure $3 \mathrm{c}$ shows a significant increase in total cells in the HF diet-fed group compared with control animals, with macrophages/monocytes constituting the majority of the recruited cells. It is noteworthy that a small number of neutrophilic-polymorphonucleated cells (PMNs) were also consistently observed, representing a statistically significant increase over those found in control animals. The number of lymphocytes was also higher in the lungs of HF diet-fed animals but the increase was not statistically significant (Figure 3b). Interestingly, a number of epithelial cells were found in BAL fluid, suggesting that some of these cells might have been sloughed off as a result of the inflammation (Figure 3b). Control animals showed a small number of monocytes/macrophages, which is consistent with the low detected levels of inflammatory cytokines (Figure 2).

\section{High-Fat Diet-Induced Lung Inflammation is Associated with Tissue Remodeling and Activation of Matrix Metalloproteinase in $\mathrm{ApoE}^{-/-}$Mice without Mucus Production}

The observed peri-vascular and peri-bronchial inflammatory cell recruitment in $\mathrm{HF}$ diet-fed $\mathrm{ApoE}^{-/-}$mice is suggestive of chronic inflammation. The persistence of inflammatory factors within lungs is known to induce tissue remodeling. To this end, we determined whether HF diet-induced inflammation was associated with changes in lung integrity.
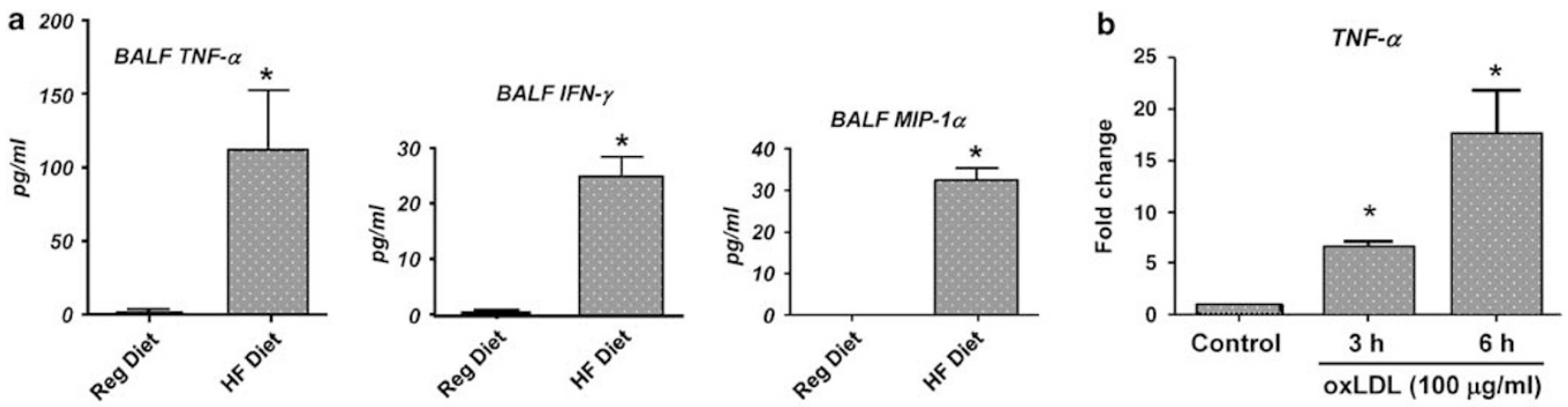

Figure 2 High-fat (HF) diet induces production of TNF- $\alpha$, IFN- $\gamma$ and MIP- $1 \alpha$ in airways of ApoE ${ }^{-/-}$mice. (a) ApoE ${ }^{-/-}$mice fed either high-fat (HF diet) or regular (Reg Diet) diet for 12 weeks were killed then subjected to brochoalveolar lavage (BAL). BAL fluids were collected and centrifuged. The supernatant were assessed for cytokine levels using the Bio-Rad Bioplex system. Data are given as means \pm s.d. of values obtained from at least six mice per group. ${ }^{*}$ Difference from $\mathrm{ApoE}^{-1-}$ mice fed a regular diet; $P<0.01$. Please note that the graphs are based on different scales. (b) Lung smooth muscle cells (SMCs) were treated with $100 \mu \mathrm{g} / \mathrm{ml}$ oxidized cholesterol (oxLDL) for the indicated time intervals, after which RNA was extracted and subjected to cDNA generation. Generated CDNA was assayed by real-time PCR with primers specific to mouse TNF- $\alpha$ or $\beta$-actin. Data are expressed as fold increase over values from untreated cells. ${ }^{*}$ Difference from untreated cells; $P<0.05$. 

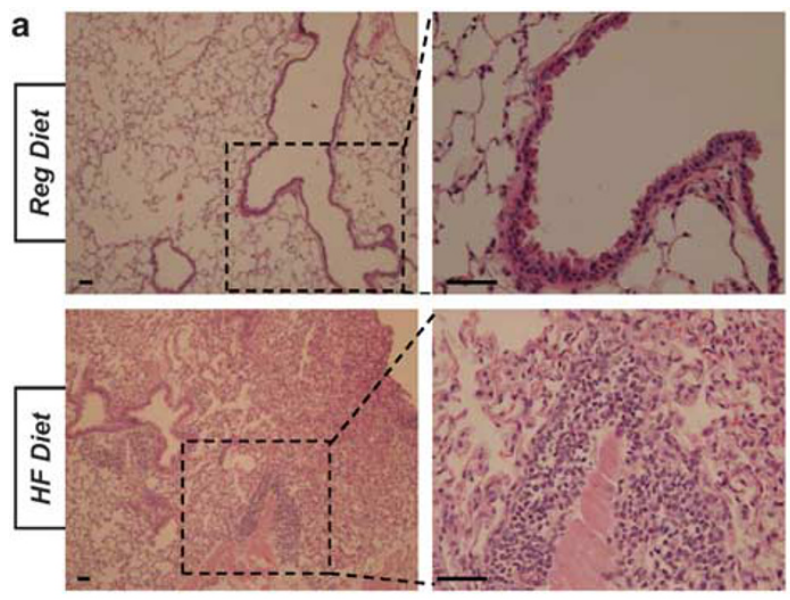

b
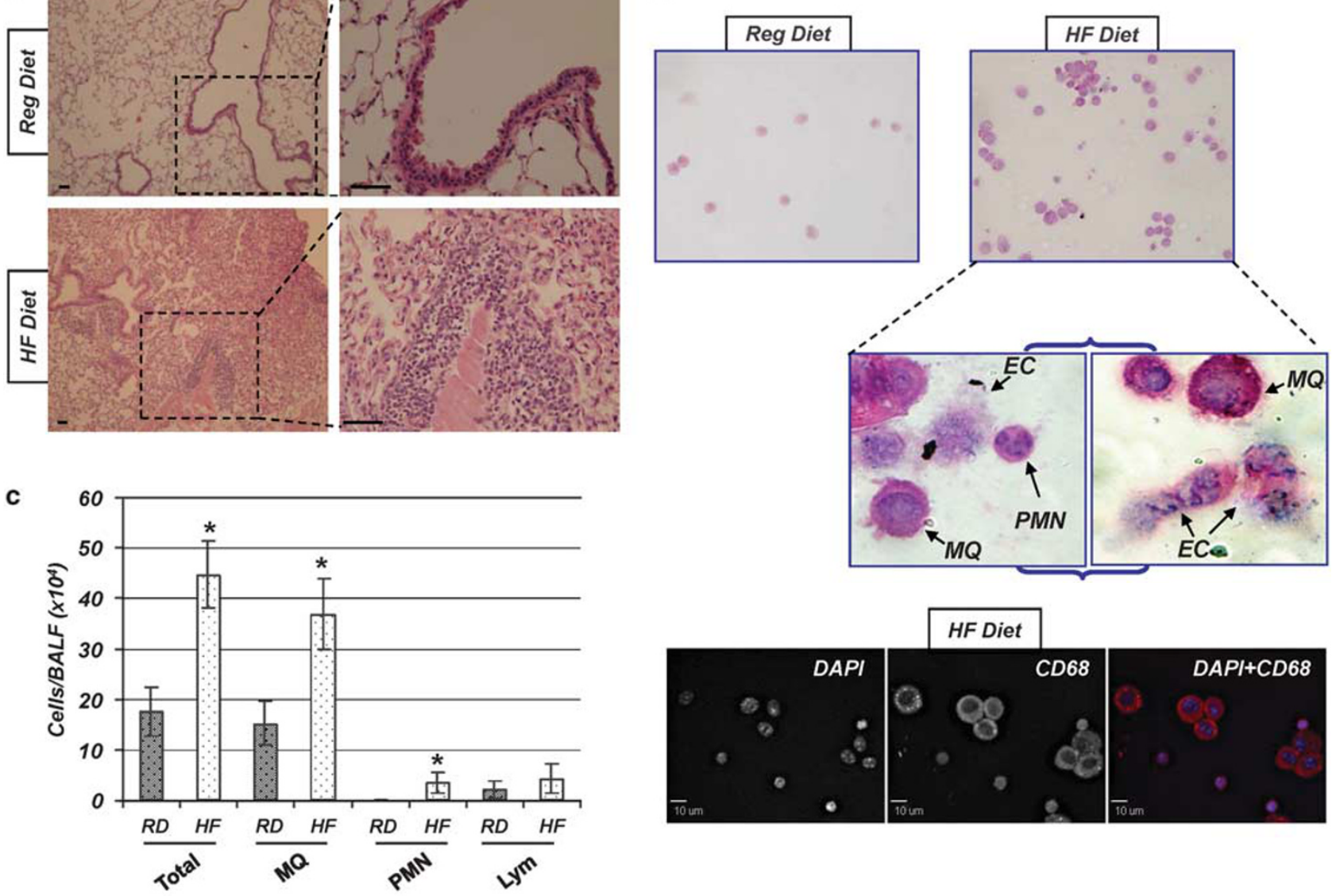

Figure 3 Association between presence of pro-inflammatory cytokines in brochoalveolar lavage (BAL) fluids from high-fat (HF) diet-fed ApoE ${ }^{-/-}$mice and airway inflammation consisting primarily of mococytes/macrophages. ApoE ${ }^{-1-}$ mice fed either high-fat (HF diet) or regular (Reg Diet) diet for 12 weeks were killed. Lungs were either fixed with formalin or subjected to BAL. (a) Lung sections were stained with H\&E and analyzed by light microscopy; bars: $5 \mu$ m. Arrows indicate peri-bronchial and peri-vascular inflammation. BAL fluids were collected and centrifuged; cells were subjected to hematoxylin and eosin (H\&E) or to immunofluorescence with antibodies to murine CD68 (b). H\&E-stained cells were also counted (c). Data are means \pm s.d. of values from at least six mice per group. ${ }^{\star}$ Difference from $\mathrm{ApoE}^{-/-}$mice fed a regular diet; $P<0.01$. Abbreviations: EC, epithelial cell; $\mathrm{MQ}$, macrophages; $\mathrm{PMN}$,

polymorphonucleated cell.

Figure 4a shows an intense trichrome staining in lungs of HF diet-fed $\mathrm{ApoE}^{-1-}$ mice, which is indicative of a robust matrix deposition. Further, matrix deposition was associated with a marked sub-epithelial and peri-vascular thickening (Figure 4a). Alveolar septae were also thickened by infiltrates of chronic inflammatory cells (data not shown).

The role of TGF- $\beta$ in lung remodeling is well established in a number of respiratory pathologies. ${ }^{18}$ Interestingly, the levels of TGF- $\beta$ in BAL fluids collected from the two experimental groups were undetectable as assessed by ELISA (data not shown). However, immunohistochemical analysis with antibodies to murine TGF- $\beta$ of lung sections of the two groups revealed a more pronounced and widespread TGF- $\beta$-immunoreactivity (Figure $4 \mathrm{~b}$ ). Although these results do not provide an unequivocal evidence for an increase in TGF- $\beta$ in the lungs of HF diet-fed mice, they do show a clear change in tissue distribution of the cytokine, which may be suggestive of a potential association between TGF- $\beta$ and HF diet- induced lung remodeling in our experimental system. Obviously, a more extensive experimentation is required for establishing or negating the role of TGF- $\beta$ in HF diet-induced lung remodeling.

A number of cytokines or growth factors, including TNF- $\alpha$ and TGF- $\beta$, trigger cell signaling, culminating in expression of MMPs. ${ }^{19}$ Protein extracts from the lungs of the two experimental groups show that an increase in MMP activity was present in the lungs of $\mathrm{ApoE}^{-1-}$ mice on the $\mathrm{HF}$ diet (Figure 4c). The $85 \mathrm{kDa}$ MMP-9 appeared to be the major member of its family activated in our experimental model. Interestingly, lung remodeling and increased MMP activity was not associated with mucus production, as assessed by PAS staining of lung sections (Figure 4d). The lack of PASpositive cells correlates with a complete absence of IL-13 production in BAL fluid of $\mathrm{HF}$ diet-fed $\mathrm{ApoE}^{-1-}$ mice (data not shown). Collectively, our results suggest that HF diet induces a chronic inflammatory response in the lungs 

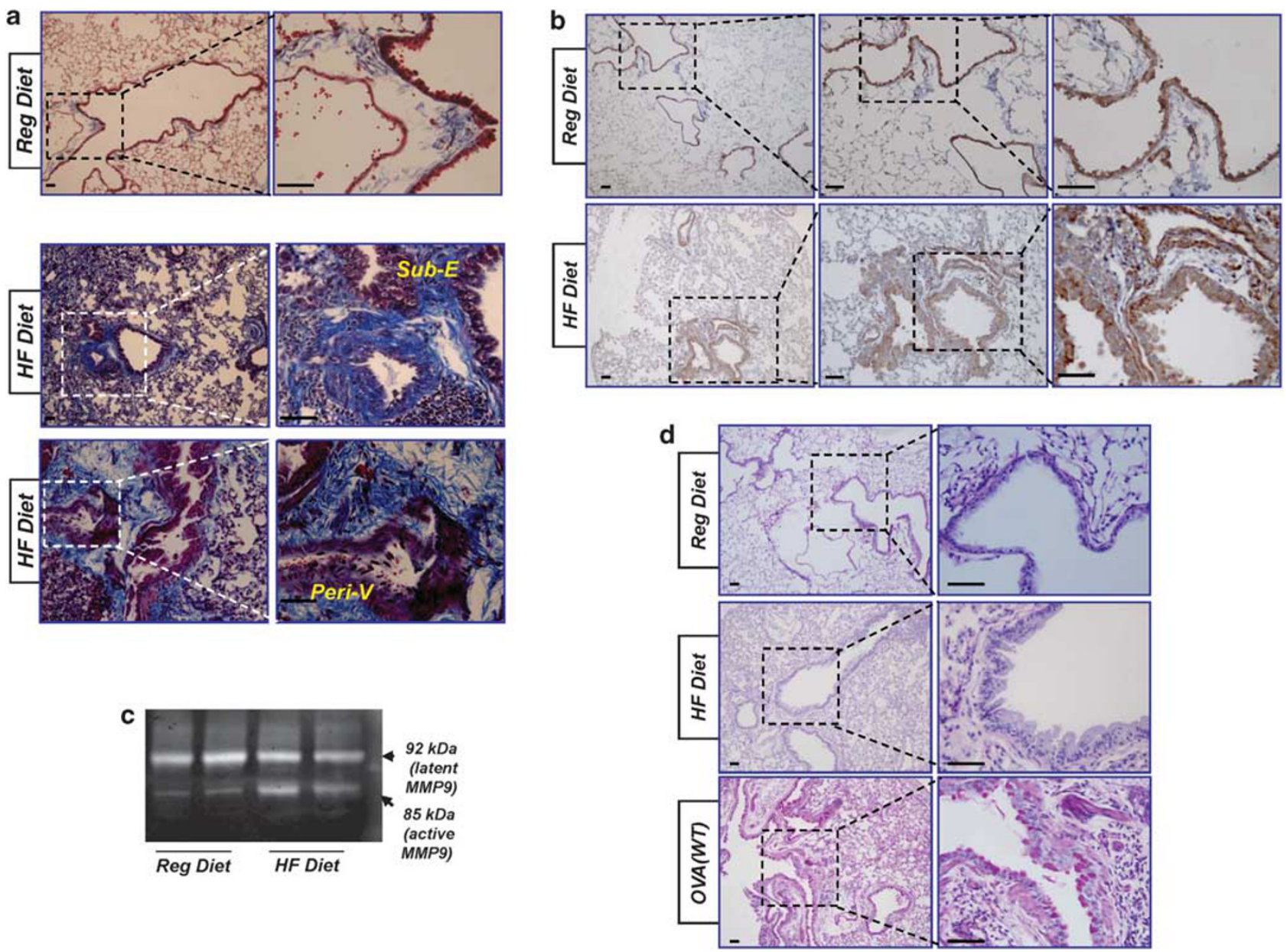

Figure 4 High-fat (HF) diet-induced airway inflammation is associated with tissue remodeling and activation of matrix metalloproteinase (MMP) in ApoE ${ }^{-1}$ - mice without an induction of mucus production. $\mathrm{ApoE}^{-/-}$mice fed either high-fat (HF diet) or regular (Reg Diet) diet for 12 weeks were killed. Lungs from these mice were then formalin-fixed for sectioning, collected for protein preparation, or subjected to brochoalveolar lavage (BAL). Lung sections were subjected to trichrome staining (a) or immunohistochemistry (IHC) with antibodies to mouse TGF- $\beta$ (b) and analyzed by light microscopy. Abbreviations: Peri-V, peri-vascular thickening; Sub-E, sub-epithelial thickening; bars: $5 \mu \mathrm{m}$. (c) Total protein $(50 \mu \mathrm{g})$ prepared from the two experimental groups was subjected to zymography as described in the Materials and methods and visualized by staining with Coomassie blue. (d) Lung sections were subjected to periodic acid-Schiff (PAS) staining. BAL fluids were assessed for IL-13 using the Bio-Rad Bioplex system. Lung sections from wild-type mice that were subjected to ovalbumin (OVA) sensitization and challenge as described ${ }^{9}$ served as controls for PAS-positive staining. ${ }^{*}$ Difference from ApoE ${ }^{-/-}$mice fed a regular diet; $P<0.01$. The right panels represent higher magnifications.

of $\mathrm{ApoE}^{-1-}$ mice, creating conditions conducive for tissue remodeling.

\section{Intratracheal TNF- $\alpha$ Administration Induces a Pattern of Lung Inflammatory Cell Recruitment as well as Expression of Key Inflammatory Factors Similar to That Observed in High-Fat Diet-Fed ApoE ${ }^{-I-}$ Mice}

As observed in Figures 1 and 2, TNF- $\alpha$ was the predominantly produced cytokine both in circulation and in the lungs of $\mathrm{ApoE}^{-1-}$ mice on a HF diet. To determine whether TNF- $\alpha$ alone can induce a pattern of inflammatory cell recruitment similar to that observed in HF diet-fed $\mathrm{ApoE}^{-1-}$ mice, wild-type mice were subjected to intratracheal administration of TNF- $\alpha$, and were killed either 6 or $24 \mathrm{~h}$ later. Lungs were removed for RNA preparation or subjected to
BAL fluid collection. It is important to note that wild-type mice were chosen for this experiment to avoid any interference by $A p o E$ gene deletion or with the associated high serum lipids. Figure 5 a shows that TNF- $\alpha$ exposure primarily induced macrophage/monocyte recruitment to the lungs, together with a small number of neutrophils. Many sloughed epithelial cells were also detected. This pathology appears to be similar to that observed in lungs of $\mathrm{ApoE}^{-1-}$ mice that were fed a HF diet for 12 weeks. Inflammatory cell recruitment into the airways in response to TNF- $\alpha$ exposure was preceded by expression of the adhesion molecules VCAM-1 and ICAM- 1 as assessed by RT-PCR of RNA from lungs $6 \mathrm{~h}$ after treatment (Figure 5b). TNF- $\alpha$ exposure also induced cytokines that are highly relevant to lung inflammation and associated remodeling, such as MCP-1, TGF- $\beta 1$, and IL- $1 \beta$ 
a
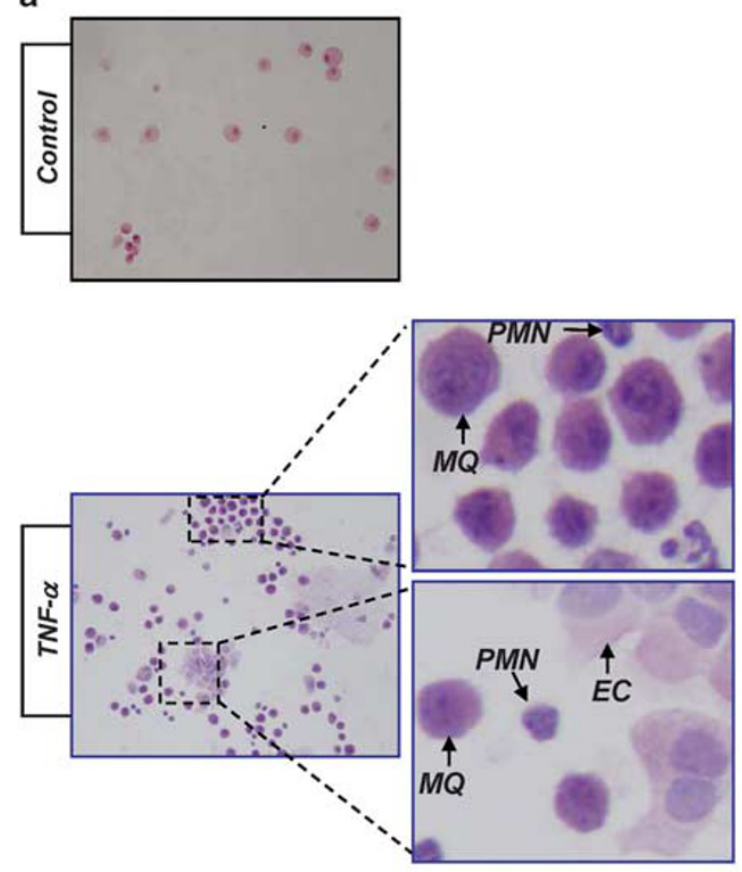

b

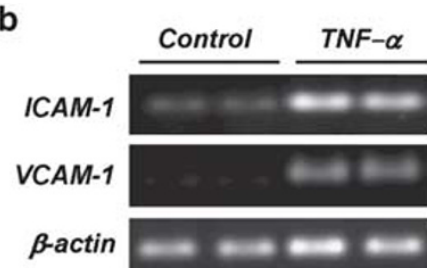

c

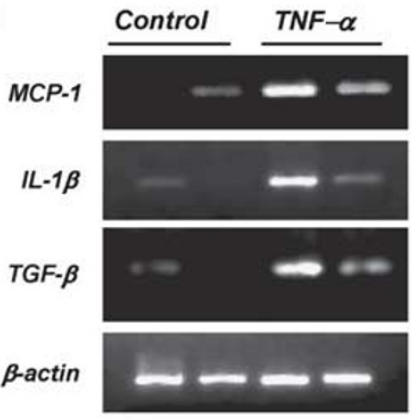

d

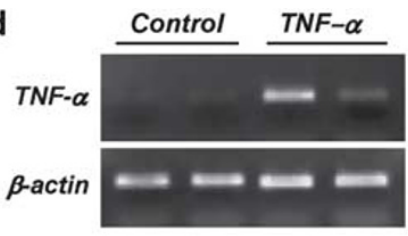

e

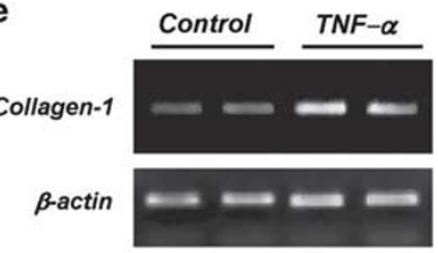

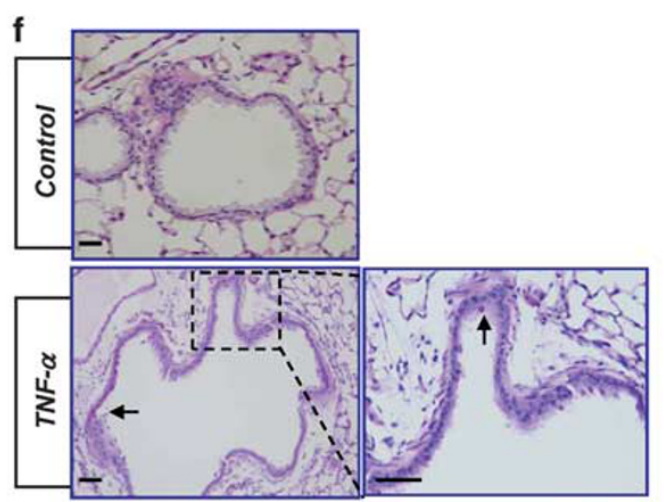

Figure 5 Intratracheal administration of TNF- $\alpha$ induces a pattern of airway inflammatory cell recruitment similar to that observed in high-fat (HF) diet-fed $\mathrm{ApoE}^{-1-}$ mice, as well as expression of key inflammatory factors. C57BL/6 wild-type mice were subjected to intranasal administration of recombinant mouse TNF- $\alpha(0.5 \mu \mathrm{g} /$ mouse) in saline or saline alone, which were then killed 6 or $24 \mathrm{~h}$ later. (a) BAL fluids were collected and centrifuged after $24 \mathrm{~h}$ treatment; cells were then differentially stained and visualized by light microscopy. Abbreviations: EC: epithelial cell; MQ: macrophage; PMN: polymorphonucleated cell. At $6 \mathrm{~h}$ after treatment, whole lungs were collected and subjected to RNA extraction followed by CDNA generation. Prepared CDNA was subjected to RT-PCR for the adhesion molecules ICAM- 1 and VCAM-1 (b), for the inflammatory factors MCP-1, IL-1- $\beta$, TGF- $\beta 1$ (c), for TNF- $\alpha$ (d), or for collagen type I (e) using their respective specific primers; $\beta$-actin was used as an internal control. (f) Formalin-fixed lung sections from control or TNF- $\alpha$-treated mice were subjected to PAS staining; arrows indicate PAS-positive epithelial cells.

(Figure $5 \mathrm{c}$ ) and TNF- $\alpha$ itself (Figure $5 \mathrm{~d}$ ), as well as collagen type I (Figure 5e). Interestingly, TNF- $\alpha$ treatment for $24 \mathrm{~h}$ did not promote a striking production of mucus as assessed by PAS staining of lung sections. These results suggest that TNF$\alpha$ may be an important player in HF diet-induced lung inflammation and associated lung remodeling in $\mathrm{ApoE}^{-1-}$ mice.

\section{DISCUSSION}

This study examines a critical question as to whether a HF diet-induced chronic inflammatory condition such as atherosclerosis influences changes within the lungs leading to important pathologies. The results of this study raise an important question as to whether chronic inflammation in cardiovascular diseases participate in the onset of lung pathological conditions such as COPD, respiratory distress syndrome or other inflammatory respiratory diseases in humans. Undoubtedly, this study is not sufficient to fully establish the effect of HF diet on lung integrity, and further experimental study is required.

The direct role of TNF- $\alpha$ in atherogenesis is well documented (reviewed by Tedgui and Mallat ${ }^{20}$ ). Circulating TNF- $\alpha$ levels are associated with increased risks of developing atherosclerosis in humans. ${ }^{20,21}$ TNF- $\alpha$ inhibition promotes a marked reduction in atherogenesis in mice fed HF diet. ${ }^{22,23}$ IFN- $\gamma$, GMC-SF, RANTES, IL- $1 \alpha$, IL- 2 and IL- 12 were detected in the sera of HF diet-fed animals in our study. The role of TNF- $\alpha$ as well as the other cytokines and chemokines we detected are well documented in lung remodeling (reviewed by de Boer et al. ${ }^{24}$ ). TNF- $\alpha$ production has been extensively associated with lung injury both in acute as well as in chronic conditions. For example, TNF- $\alpha$-deficient mice 
are resistant to acute lung injury in response to LPS treatment. ${ }^{25}$ Whether the circulatory TNF- $\alpha$ itself leaked into the lungs or was produced by lung cells is not clear. We do know that intratracheal administration of TNF- $\alpha$ in wild-type mice induced the expression of several inflammatory factors that are important for inflammatory cell recruitment, such as the adhesion molecules ICAM- 1 and VCAM-1, and the chemotactic cytokine MCP- 1 , IL- $1 \beta$ and TGF- $\beta$, TNF- $\alpha$ itself, as well as collagen type I, all of which are known to contribute to lung injury and lung remodeling. The similarities between the nature of the inflammatory cells that were recruited to the lungs in $\mathrm{HF}$ diet-fed $\mathrm{ApoE}^{-1-}$ mice and in TNF- $\alpha$-treated wild-type mice were striking. It is noteworthy that the antiinflammatory cytokine IL-10 was also elevated in the sera of HF diet-fed animals. Given the pathological changes elicited by HF diet, it would be plausible to consider that the conditions in our experimental model are tilted toward a proinflammatory state.

Elevated TNF- $\alpha$ levels and lung inflammation in the HF diet-fed mice also coincided with an increase in MMP-9 activity. MMP-9 enzymatic activity is one of the most important proteases involved in lung remodeling associated with respiratory diseases such as COPD. TNF- $\alpha$ is crucial for MMP-9 expression ${ }^{26}$ and may be associated with the increased activity of MMP-9 in response to the HF diet in $\mathrm{ApoE}^{-1-}$ mice. Indeed, Lai et al. ${ }^{27}$ have recently reported that TNF- $\alpha$ not only increases expression of MMP- 9 but also promotes its activation in aortic vascular SMCs and in mice. Such increase in MMP-9 expression and activity has also been reported upon exposure to TGF- $\beta$ in cultured cells. ${ }^{28}$ Tissue remodeling and expression of MMPs are closely related to TGF- $\beta$ production and associated signal transduction. ${ }^{29}$ It was rather surprising that HF diet did not induce a detectable production of TGF- $\beta$ in BAL fluids of $\mathrm{ApoE}^{-/-}$ mice but a change in its tissue distribution compared with that observed in control mice was apparent. It is difficult to attribute this change to the observed tissue remodeling induced by HF diet, but it is clear that the diet promoted factors that caused the change in distribution. TNF- $\alpha$ induced expression of TGF- $\beta$ in lungs of wild-type mice, which is consistent with a recent report by Brody's group. ${ }^{30}$ The reason behind the failure of HF diet to induce production of TGF- $\beta$ in $\mathrm{ApoE}^{-/-}$mice is not clear.

An important issue worth mentioning is the fact that $\mathrm{ApoE}^{-1-}$ mice that are fed a regular diet show serum cholesterol levels that are two to three times the levels of wildtype mice. The question of why these mice did not show any signs of lung remodeling or increased cytokines in the sera is valid. The lack of such an effect could be attributed to the short treatment duration. It is noteworthy that $\mathrm{ApoE}^{-1-}$ mice eating a regular diet can develop atherosclerotic plaques similar to those observed when given a HF diet, but time to development is far longer than with the HF diet. Accordingly, it is tempting to speculate that $\mathrm{ApoE}^{-/-}$mice could develop lung remodeling while on a regular diet, if given sufficient time. However, this hypothesis remains to be tested. Interestingly, Massaro and Massaro ${ }^{31}$ reported that $\mathrm{ApoE}^{-1-}$ mice showed serious defects in lung function, including increased lung resistance in early adulthood and high lung volume and high dynamic and static compliance in later adulthood compared with wild-type mice. They predicted that dietary and/or genetic effects on lipid metabolism might be an upstream cause of inflammation and oxidative stress contributing to lung pathologies such as those associated with COPD. It is important to note that the role of ApoE lipoprotein in preventing and/or modulating inflammation is well documented (reviewed by Jofre-Monseny et al. ${ }^{32}$ ). For instance, ApoE protein or ApoE mimetic peptides have been shown to suppress cellular responses to LPS, ${ }^{33}$ microglial activation and release of TNF- $\alpha,{ }^{34}$ as well as modulate rapidly evolving atherosclerotic lesions in vein grafts. ${ }^{35}$ Whether ApoE deficiency has a direct role in the lung remodeling observed in our study remains to be determined. However, although our in vitro data does exclude a role for ApoE in lung inflammation, they show that direct exposure of lung cells to oxLDLs culminates in the production of TNF- $\alpha$, which are the primary cytokines observed in our animal model both systemically and in the lungs of HF diet-fed animals.

Supplementary Information accompanies the paper on the Laboratory Investigation website (http://www.laboratoryinvestigation.org)

\section{ACKNOWLEDGEMENTS}

We thank Dr K Espinoza for her assistance with pathology. We also thank Ji-Won Park and Waleed Elsegeiny for their technical assistance. This work was supported in part by grants HL072889 from the National Institute of Health and funds from the Louisiana Cancer Research Consortium (New Orleans, LA, USA) to H Boulares.

\section{DISCLOSURE/CONFLICT OF INTEREST}

The authors declare no conflict of interest.

1. Innis SM. Dietary lipids in early development: relevance to obesity, immune and inflammatory disorders. Curr Opin Endocrinol Diabetes Obes 2007;14:359-364.

2. Libby P. Inflammation and cardiovascular disease mechanisms. Am J Clin Nutr 2006;83:456S-460S.

3. Gern JE, Brockman-Schneider R, Bhattacharya S, et al. Serum and lowdensity lipoprotein enhance interleukin- 8 secretion by airway epithelial cells. Am J Respir Cell Mol Biol 2003;29:483-489.

4. Yeh YF, Huang SL. Dietary cholesterol enhances pulmonary eosinophilic inflammation in a murine model of asthma. Int Arch Allergy Immunol 2001;125:329-334.

5. Al-Shawwa B, Al-Huniti N, Titus G, et al. Hypercholesterolemia is a potential risk factor for asthma. J Asthma 2006;43:231-233.

6. Maddox L, Schwartz DA. The pathophysiology of asthma. Annu Rev Med 2002;53:477-498.

7. Broide $D$. New perspectives on mechanisms underlying chronic allergic inflammation and asthma in 2007. J Allergy Clin Immunol 2008; 122:475-480.

8. Oumouna M, Datta R, Oumouna-Benachour K, et al. PARP-1 inhibition prevents eosinophil recruitment by modulating Th2 cytokines in a murine model of allergic airway inflammation: a potential specific effect on IL-5. J Immunol 2006;177:6489-6496.

9. Naura AS, Hans CP, Zerfaoui M, et al. Post-allergen challenge inhibition of poly(ADP-ribose) polymerase harbors therapeutic potential for treatment of allergic airway inflammation. Clin Exp Allergy 2008;38:839-846. 
10. Oumouna-Benachour K, Hans CP, Suzuki Y, et al. Poly(ADP-ribose) polymerase inhibition reduces atherosclerotic plaque size and promotes factors of plaque stability in apolipoprotein E-deficient mice: effects on macrophage recruitment, nuclear factor-kappaB nuclear translocation, and foam cell death. Circulation 2007;115: 2442-2450.

11. Boulares AH, Zoltoski AJ, Sherif ZA, et al. Gene knockout or pharmacological inhibition of poly(ADP-ribose) polymerase-1 prevents lung inflammation in a murine model of asthma. Am J Respir Cell Mol Biol 2003;28:322-329.

12. Amrani $\mathrm{Y}$, Lazaar AL, Hoffman R, et al. Activation of $\mathrm{p} 55$ tumor necrosis factor-alpha receptor- 1 coupled to tumor necrosis factor receptor-associated factor 2 stimulates intercellular adhesion molecule- 1 expression by modulating a thapsigargin-sensitive pathway in human tracheal smooth muscle cells. Mol Pharmacol 2000:58:237-245.

13. Oumouna-Benachour K, Oumouna M, Zerfaoui $M$, et al. Intrinsic resistance to apoptosis of colon epithelial cells is a potential determining factor in the susceptibility of the $A / J$ mouse strain to dimethylhydrazine-induced colon tumorigenesis. Mol Carcinog 2007;46:993-1002.

14. Zerfaoui $M$, Suzuki $Y$, Naura AS, et al. Nuclear translocation of $p 65$ NF-kappaB is sufficient for VCAM-1, but not ICAM-1, expression in TNF-stimulated smooth muscle cells: differential requirement for PARP-1 expression and interaction. Cell Signal 2008;20:186-194.

15. Boulares AH, Zoltoski AJ, Yakovlev A, et al. Roles of DNA fragmentation factor and Poly(ADP-ribose) polymerase in an amplification phase of tumor necrosis factor-induced apoptosis. J Biol Chem 2001;276:38185-38192.

16. Libby P. Atherosclerosis: disease biology affecting the coronary vasculature. Am J Cardiol 2006;98:3Q-9Q.

17. Vignola AM, Mirabella F, Costanzo G, et al. Airway remodeling in asthma. Chest 2003;123(Suppl):417S-422S.

18. Koli K, Myllarniemi M, Keski-Oja J, et al. Transforming growth factorbeta activation in the lung: focus on fibrosis and reactive oxygen species. Antioxid Redox Signal 2008;10:333-342.

19. Yan C, Boyd DD. Regulation of matrix metalloproteinase gene expression. J Cell Physiol 2007;211:19-26.

20. Tedgui A, Mallat Z. Cytokines in atherosclerosis: pathogenic and regulatory pathways. Physiol Rev 2006;86:515-581.

21. Kleemann R, Zadelaar S, Kooistra T. Cytokines and atherosclerosis: a comprehensive review of studies in mice. Cardiovasc Res 2008; 79:360-376.
22. Branen L, Hovgaard L, Nitulescu M, et al. Inhibition of tumor necrosis factor-alpha reduces atherosclerosis in apolipoprotein $E$ knockout mice. Arterioscler Thromb Vasc Biol 2004:24:2137-2142.

23. Elhage R, Maret A, Pieraggi MT, et al. Differential effects of interleukin-1 receptor antagonist and tumor necrosis factor binding protein on fatty-streak formation in apolipoprotein E-deficient mice. Circulation 1998;97:242-244.

24. de Boer WI, Yao H, Rahman I. Future therapeutic treatment of COPD: struggle between oxidants and cytokines. Int J Chron Obstruct Pulmon Dis 2007;2:205-228.

25. Okamoto T, Gohil K, Finkelstein El, et al. Multiple contributing roles for NOS2 in LPS-induced acute airway inflammation in mice. Am J Physiol Lung Cell Mol Physiol 2004;286:L198-L209.

26. Lin CC, Tseng HW, Hsieh HL, et al. Tumor necrosis factor-alpha induces MMP-9 expression via p42/p44 MAPK, JNK, and nuclear factor-kappaB in A549 cells. Toxicol Appl Pharmacol 2008;229:386-398.

27. Lai CF, Seshadri V, Huang $K$, et al. An osteopontin-NADPH oxidase signaling cascade promotes pro-matrix metalloproteinase 9 activation in aortic mesenchymal cells. Circ Res 2006;98:1479-1489.

28. Richiert DM, Ireland ME. Matrix metalloproteinase secretion is stimulated by TGF-beta in cultured lens epithelial cells. Curr Eye Res 1999;19:269-275.

29. Verrecchia F, Mauviel A. Transforming growth factor-beta and fibrosis. World J Gastroenterol 2007;13:3056-3062.

30. Sullivan $D E$, Ferris $M$, Pociask $D$, et al. Tumor necrosis factor-alpha induces transforming growth factor-beta1 expression in lung fibroblasts through the extracellular signal-regulated kinase pathway. Am J Respir Cell Mol Biol 2005;32:342-349.

31. Massaro D, Massaro GD. Apoetm1Unc mice have impaired alveologenesis, low lung function, and rapid loss of lung function. Am J Physiol Lung Cell Mol Physiol 2008;294:L991-L997.

32. Jofre-Monseny L, Minihane AM, Rimbach G. Impact of apoE genotype on oxidative stress, inflammation and disease risk. Mol Nutr Food Res 2008;52:131-145.

33. Berbee JF, Havekes LM, Rensen PC. Apolipoproteins modulate the inflammatory response to lipopolysaccharide. J Endotoxin Res 2005;11:97-103.

34. Laskowitz DT, Thekdi AD, Thekdi SD, et al. Downregulation of microglial activation by apolipoprotein $\mathrm{E}$ and apoE-mimetic peptides. Exp Neurol 2001;167:74-85.

35. Li X, Chyu KY, Faria Neto JR, et al. Differential effects of apolipoprotein A-I-mimetic peptide on evolving and established atherosclerosis in apolipoprotein E-null mice. Circulation 2004;110:1701-1705. 